\title{
Test of QCD in Multijet Final States at the Tevatron
}

\author{
Ashish Kumar \\ The State University of New York at Buffalo, NY 14260, USA \\ (on behalf of the CDF and D0 Collaborations)
}

\begin{abstract}
In this contribution, some of the recent QCD results on jet production from the CDF and D0 experiments in Run II of the Tevatron are discussed. In particular, results include cross section measurements for dijet and trijet production, study of jet substructure and measurements of W/Z+jets cross sections. The measurements are compared with the theoretical predictions.
\end{abstract}

Keywords: Jet Production, Vector Boson plus Jet Production

PACS: 13.87.-a

\section{INTRODUCTION}

The QCD program at the Tevatron proton-antiproton collider revolves around the jet physics. Jets are collimated sprays of hadrons generated by the fragmentation of partons originating from the hard scattering. The measurements of jets production cross section at the Tevatron provide stringent test of perturbative QCD predictions, information on the strong coupling constant, $\alpha_{s}$, and constraints on proton parton distribution functions, PDFs. The deviations from the PQCD predictions could translate into a hint of new physics. In addition, these processes form sizable backgrounds to both, the Standard Model (SM) and beyond SM physics processes. Therefore, good understanding of jets production is essential to perform precise physics measurements or searches for new physics phenomena.

In this contribution, a review of some recent QCD results from the CDF [1] and D0 [2] experiments is presented. Various analyses use proton-antiproton collision data from the Fermilab Tevatron at the center of mass energy $\sqrt{s}=1.96 \mathrm{TeV}$ corresponding to integrated luminosities of $0.7 \mathrm{fb}^{-1}$ to $8 \mathrm{fb}^{-1}$. The results are corrected for the experimental effects and presented at the "particle level". The theory predictions are corrected for the hadronization and underlying effects which are obtained from the parton shower Monte Carlo (MC) simulations.

\section{RESULTS}

\section{Dijet Production}

The D0 collaboration has measured the inclusive dijet double differential cross section as a function of the dijet invariant mass $\left(\mathrm{M}_{\mathrm{jj}}\right)$ and of the largest absolute rapidity $\left(|\mathrm{y}|_{\max }\right)$ of the two jets with the largest transverse momentum $\mathrm{p}_{\mathrm{T}}$ in an event using $0.7 \mathrm{fb}^{-1}$ of data [3]. Both jets are required to have $\mathrm{p}_{\mathrm{T}}>40 \mathrm{GeV}$. The measurement has been done in six bins of $|y|_{\max }$ for $0<|y|_{\max }<2.4$. The dijet mass cross section is presented in Fig. 1(a). In general, the data are described by next-to-leading order (NLO) pQCD calculations using MSTW2008 or CTEQ6.6 PDFs in all rapidity regions, though the central value of the CTEQ6.6 PDFs differs from data for high dijet mass at larger rapidities. The CDF collaboration has searched for narrow mass resonances in the dijet mass distribution requiring jet rapidities $|y|<1$ [4]. Both experiments demonstrate a mass reach up to $1.2 \mathrm{TeV}$ and test pQCD predictions, with no indications of resonances as signatures of physics beyond the SM.

\section{Trijet Production}

Three jet production provides a further test of QCD which is less dependent on the parameterization of PDFs, and in principle directly probes the $\alpha_{\mathrm{s}}$. Using an integrated luminosity of $0.7 \mathrm{fb}^{-1}$, the D0 Collaboration has presented first measurement of the inclusive three-jet differential cross section as a function of the invariant three-jet mass $\left(\mathrm{M}_{3 \mathrm{jet}}\right)$ reaching masses up to $1.1 \mathrm{TeV}$ [5]. The events are required to have $\mathrm{p}_{\mathrm{T}}$ of the leading jet larger than $150 \mathrm{GeV}$ and for 
any pair of jets to be well separated in $\eta-\phi$ space. The measurement is performed in three rapidity regions $(|y|<2: 4 ; 1: 6 ; 0: 8)$ and in three regions of the third jet $\mathrm{p}_{\mathrm{T}}(40 ; 70 ; 100 \mathrm{GeV})$. The comparison of the experimental data with the NLO theoretical predictions shows a reasonable agreement and favor MSTW2008 and NNPDF2.1 PDF sets (see Fig. 1(b)).

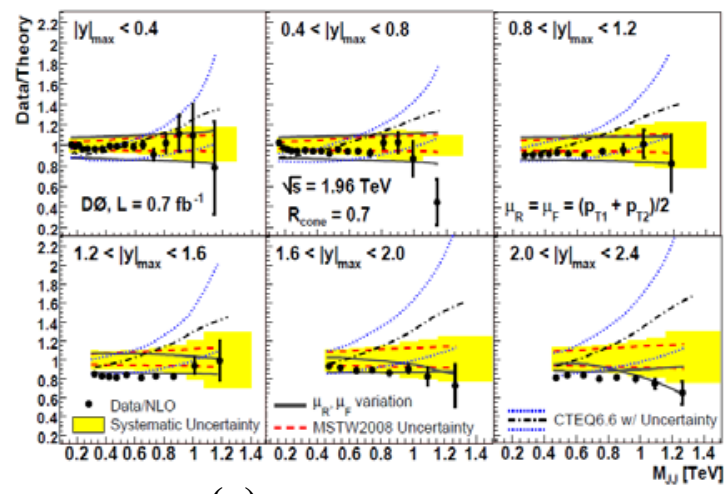

(a)

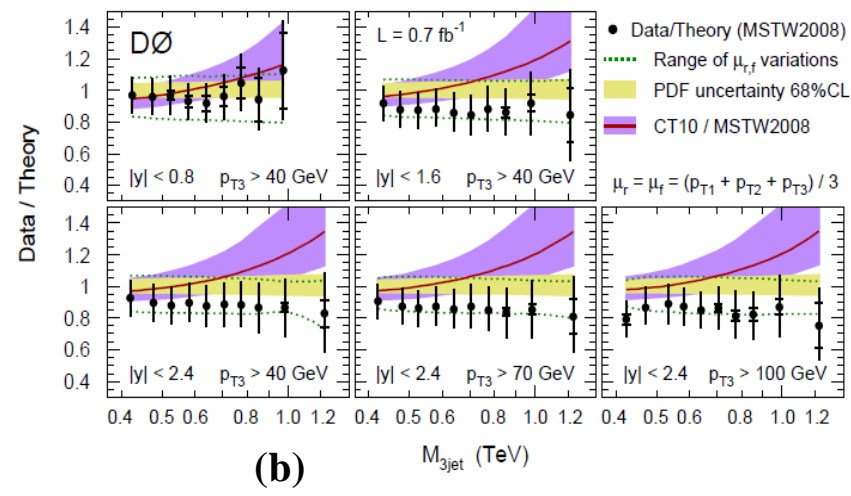

(b)

FIGURE 1. (a) Measured dijet production cross section as a function of the dijet invariant mass, Mjj, divided by the NLO QCD prediction. (b) The data over theory ratio for the three-jet invariant mass cross section showing the range of the scale variation (green dotted lines) and MSTW2008NLO PDF uncertainty (yellow band.)

\section{Study of Substructure of High $p_{T}$ Jets}

The study of high $\mathrm{p}_{\mathrm{T}}$ massive jets provides an important test of PQCD and gives insight into the parton showering mechanism. In addition, massive boosted jets comprise an important background in searches for various new physics processes, the Higgs boson, and highly boosted top quark pair production. Particularly relevant is the case where the decay of a heavy resonance produces high- $\mathrm{p}_{\mathrm{T}}$ top quarks that decay hadronically. In all these cases, the hadronic decay products can be detected as a single jet with substructure that differs from QCD jets once the jet $\mathrm{p}_{\mathrm{T}}$ is greater than 400-500 GeV. Using $6 \mathrm{fb}^{-1}$ data, the CDF has performed the first measurement of substructure of jets [6] with $\mathrm{p}_{\mathrm{T}}>400 \mathrm{GeV}$ by studying distributions of the jet mass and measuring "angularity", the variable describing the energy distribution inside the jet, and "planar flow", the variable differentiating between two-prong and three-prong decays. At small cone sizes and large jet mass, these variables are expected to be quite robust against soft radiation and allow, in principle, a comparison with theoretical predictions.
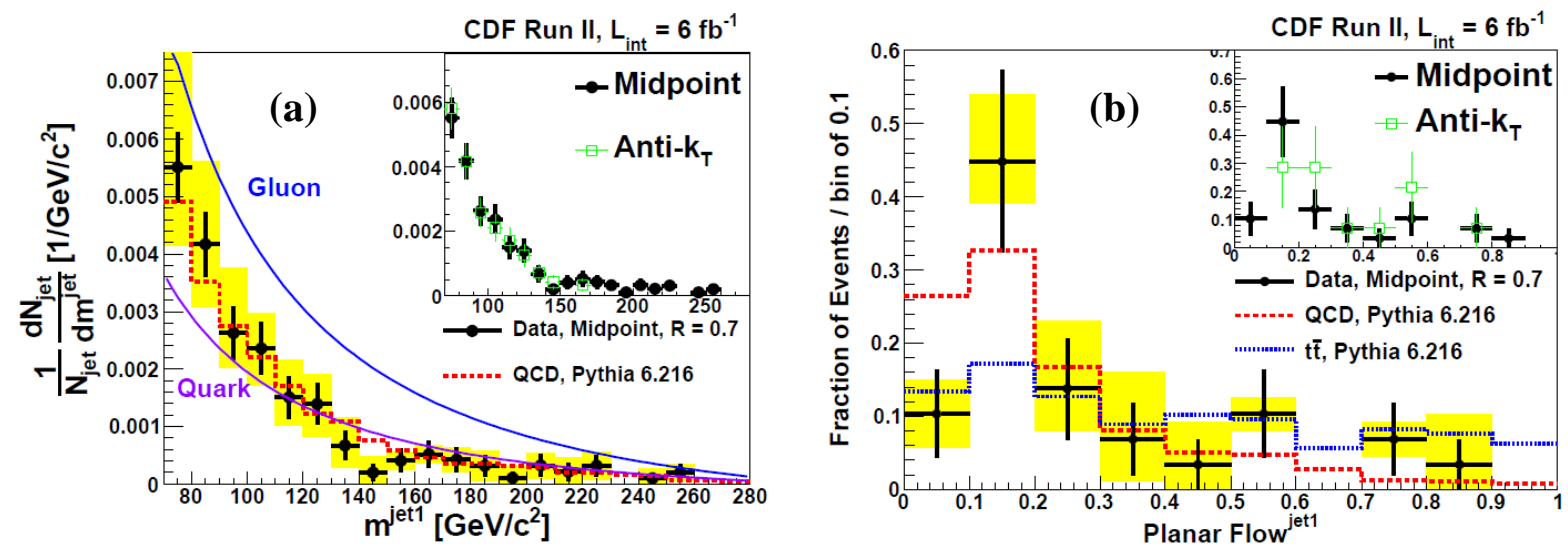

FIGURE 2. (a) The normalized jet mass distribution for midpoint jets with $\mathrm{p}_{\mathrm{T}}>400 \mathrm{GeV}$. The theory predictions for the jet functions for quarks and gluons are shown as solid curves and have an estimated uncertainty of 30\%. Also shown is the PYTHIA MC prediction. The inset compares midpoint and anti-kt jets. (b) The planar fow distributions after applying the top rejection cuts and requiring $130 \mathrm{GeV}<\mathrm{m}^{\text {jet1 }}<210 \mathrm{GeV}$. PYTHIA QCD (red dashed line) and top pair (blue dotted line) jets are also shown. 
Figure 2(a) shows that the measured jet mass distribution is in good agreement with the analytic predictions for the jet function and with PYTHIA predictions. It has been found that the midpoint and anti-kt algorithms have very similar jet substructure distributions of high mass jets. The results have also shown that the high mass jets coming from light quark and gluon fragmentation are consistent with two-body final states from a study of the angularity variable, and that further rejection against high mass QCD jets can be obtained using the planar flow variable (see Fig 2(b).

\section{W/Z + Jets Production}

Study of the associated production of vector bosons with jets constitutes an important topic in the high-p physics program at the Tevatron. Measurements of production cross sections and kinematic distributions of W/Z+jets provide a stringent test of QCD predictions at high $\mathrm{Q}^{2}$ and offer the possibility to validate MC simulation tools. Furthermore, W/Z+jets processes constitute a significant source of background in many measurements and searches at the Tevatron and the LHC, like top-quark measurements and Higgs boson and super-symmetric particles searches.

The CDF experiment has measured the $\mathrm{Z} / \gamma^{*}+$ jets production cross sections in the dielectron and dimuon decay channels of $\mathrm{Z}$ with about $6 \mathrm{fb}^{-1}$ of data [7][8]. Differential cross sections as a function of $\mathrm{p}_{\mathrm{T}}^{\text {jet }}$, $|\mathrm{y}|^{\text {jet }}$, and jet multiplicity, have been measured. Events are required to have two opposite signed electrons or muons with a reconstructed invariant mass in the range $66<\mathrm{M}_{\mathrm{Z}}<116 \mathrm{GeV}$ around the $\mathrm{Z}$ boson mass. Jets are clustered with the Midpoint algorithm in a cone radius of 0.7 , and are required to have $\mathrm{p}_{\mathrm{T}}>30 \mathrm{GeV}$ and $|\mathrm{y}| \leq 2.1$. The data is compared to NLO pQCD predictions obtained with the MCFM program. Figure 3(a) shows the measured inclusive differential cross section in $\mathrm{Z}+\geq 1$ jet events. Figure 3(b) shows the measured cross section as a function of jet multiplicity compared to LO and NLO PQCD predictions obtained with MCFM. The measurements are well described by the NLO predictions. For $\mathrm{N}_{\mathrm{jet}} \geq 3$, only $\mathrm{LO}$ predictions are available.
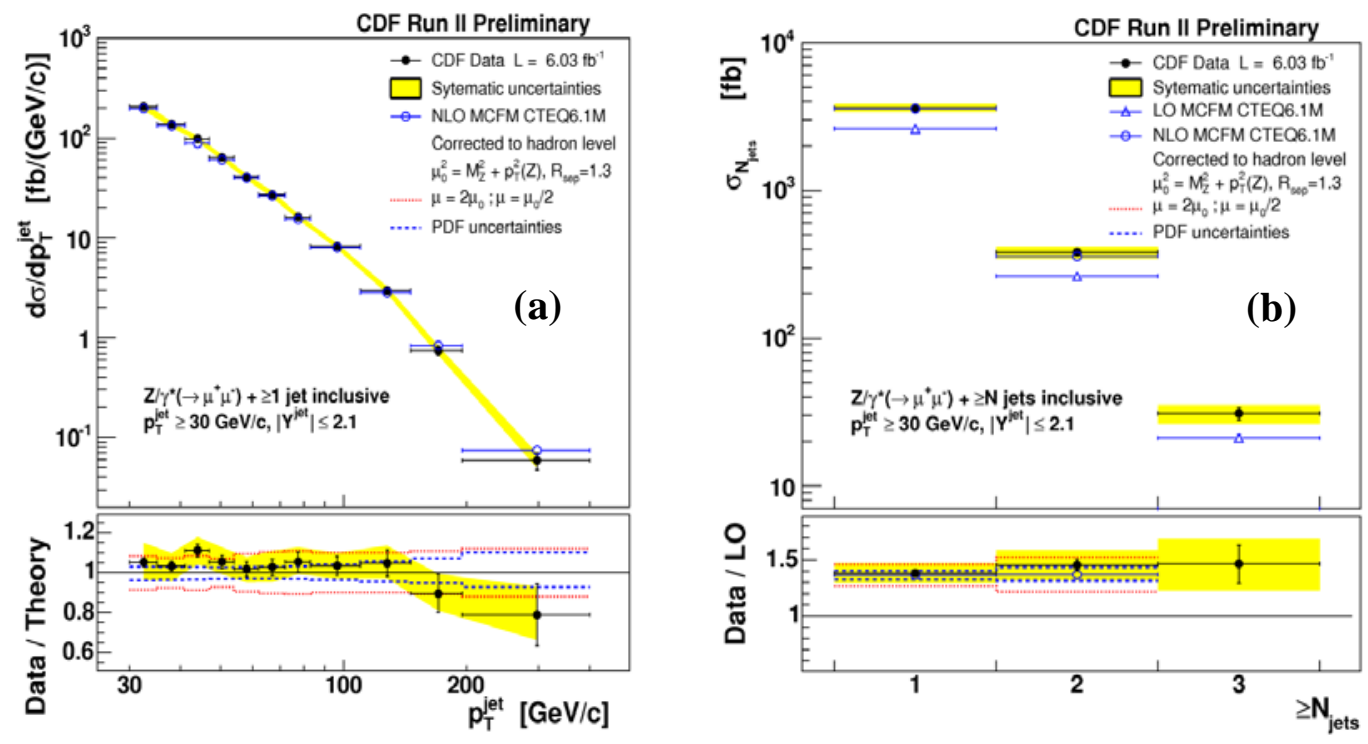

FIGURE 3. (a) Measured inclusive jet differential cross sections as a function of jet $\mathrm{p}_{\mathrm{T}}$ in $\mathrm{Z} / \gamma^{*} \rightarrow \mu^{+} \mu^{-}+\geq 1$ jet events. (b) Measured total cross section for inclusive jet production in $\mathrm{Z} / \gamma^{*} \rightarrow \mu^{+} \mu^{-}$events as a function of jet multiplicity. Data (black dots) are compared to NLO pQCD predictions (open circles). The shaded bands show the total systematic uncertainty, except for the $5.8 \%$ uncertainty on the luminosity. The dashed and dotted lines indicate the PDF uncertainty and the variation with $\mu_{0}$ of the NLO pQCD predictions, respectively.

D0 has recently presented measurements of $\mathrm{W}(\rightarrow \mathrm{ev})+\mathrm{n}$ jets cross-sections for $\mathrm{n}=1,2,3$ and 4 jets using a data sample of $4.2 \mathrm{fb}^{-1}$ [9]. The measurements include the total inclusive cross section for each jet multiplicity and differential cross sections normalized by inclusive $\mathrm{W}$ cross section as a function of $\mathrm{n}^{\text {th }}$ jet $\mathrm{p}_{\mathrm{T}}$. The measurements improve on the previous CDF measurement by including the $\mathrm{W}+4$ jet differential cross sections, and by significantly 
improving the uncertainties on the differential cross sections as well as by performing the first comparison with NLO W+3 jet predictions using BLACKHAT+SHERPA [10] and ROCKET+MCFM [11]. Figure 4 shows the ratio of the predictions to the measured differential cross sections as a function of the $\mathrm{n}^{\text {th }}$ jet $\mathrm{p}_{\mathrm{T}}$ in $\mathrm{W}+\mathrm{n}$ jet events. For the $\mathrm{W}+4$ jet production, only LO prediction is available. The measured cross sections are generally found to agree with the theory although certain regions of phase space are identified, where these predictions could better match the data.
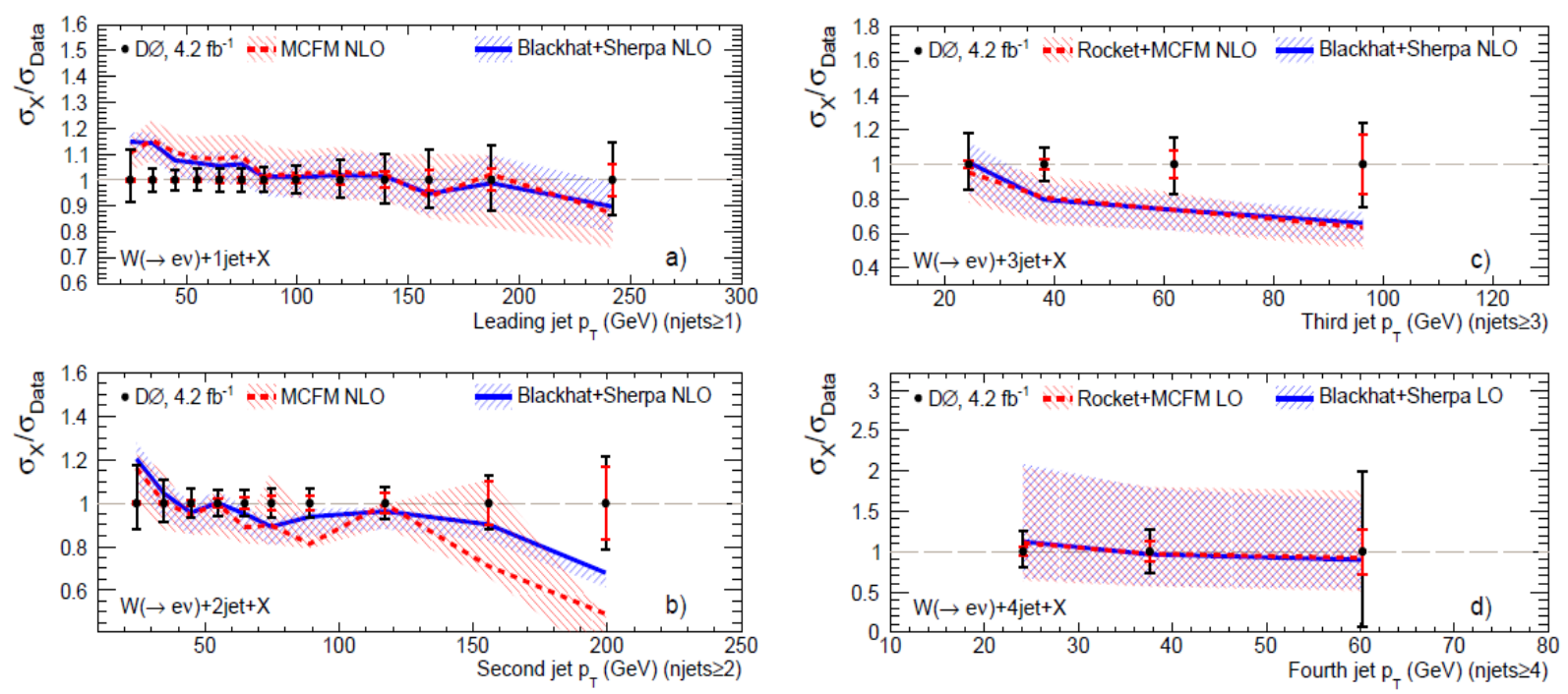

FIGURE 4. The ratio of the predictions to the measured differential cross sections as a function of the nth jet $\mathrm{p}_{\mathrm{T}}$ in (a) $\mathrm{W}+1$ jet events, (b) W+2 jet events, (c) W+3 jet events, and (d) W+4 jet events.

\section{W/Z + Heavy Flavor Jet Production}

The D0 experiment has published the most precise measurement of the ratio of cross-sections for the Z+b-jet to $\mathrm{Z}+$ jet production based on about $4.2 \mathrm{fb}^{-1}$ of data [12]. The measurement of the ratio benefits from cancellations of many systematic uncertainties, and therefore allows a more precise comparison with theory. Events that are consistent with the electronic and muonic $Z$ boson decay containing at least one jet (cone size $\mathrm{R}=0.5, \mathrm{p}_{\mathrm{T}}>20 \mathrm{GeV}$ and $|\eta|<2.5$ ) are selected. The b-jets are identified by means of a dedicated neural network (NN) technique that exploits the various characteristics of tracks of particles inside the jet. The b-jet fraction in the sample enriched with heavy flavors is extracted using a state of the art discriminant constructed from impact parameter significance of tracks associated with the jet and the secondary vertex mass. Figure 5(a) shows the discriminant distribution of btagged jets for data along with the fitted contributions from jets of different flavors. The measured ratio $\sigma(\mathrm{Z}+\mathrm{b}) / \sigma(\mathrm{Z}+$ jet$)=0.0193 \pm 0.0022$ (stat) \pm 0.0015 (syst) is in good agreement with the theoretical prediction of $0.0192 \pm 0.0022$.

In the same $\mathrm{Z}+\mathrm{b}$-jet final state, the CDF experiment has recently presented a preliminary measurement with about 8 $\mathrm{fb}^{-1}$ of integrated luminosity [13]. Jets with cone size $\mathrm{R}=0.7, \mathrm{p}_{\mathrm{T}}>20 \mathrm{GeV}$ and $|\eta|<1.5$ are selected. A $b$-tagging algorithm, based on displaced secondary vertices, is used to enhance the presence of $b$-jets in the selected sample. In this measurement the b-quark jets fraction is extracted from a fit to the mass of a displaced secondary vertex reconstructed within the jet. Figure 5(b) shows the secondary vertex mass distribution of b-tagged jets for data along with the fitted contributions from jets of different flavors. The $\mathrm{Z}+\mathrm{b}$-jet cross section is normalized to the inclusive $\mathrm{Z}$ cross section in order to reduce the systematic uncertainties, and the measured value is $\sigma(\mathrm{Z}+\mathrm{b}) / \sigma(\mathrm{Z})=2.84 \pm 0.29$ $\pm 0.29 \times 10^{-3}$ which is consistent with predicted value from MCFM. The cross section is also normalized to the $\mathrm{Z}+$ jets productions and the measured ratio $\sigma(\mathrm{Z}+\mathrm{b}) / \sigma(\mathrm{Z}+\mathrm{jet})=2.24 \pm 0.24 \pm 0.26 \times 10^{-2}$ also agrees well with the theoretical prediction. 

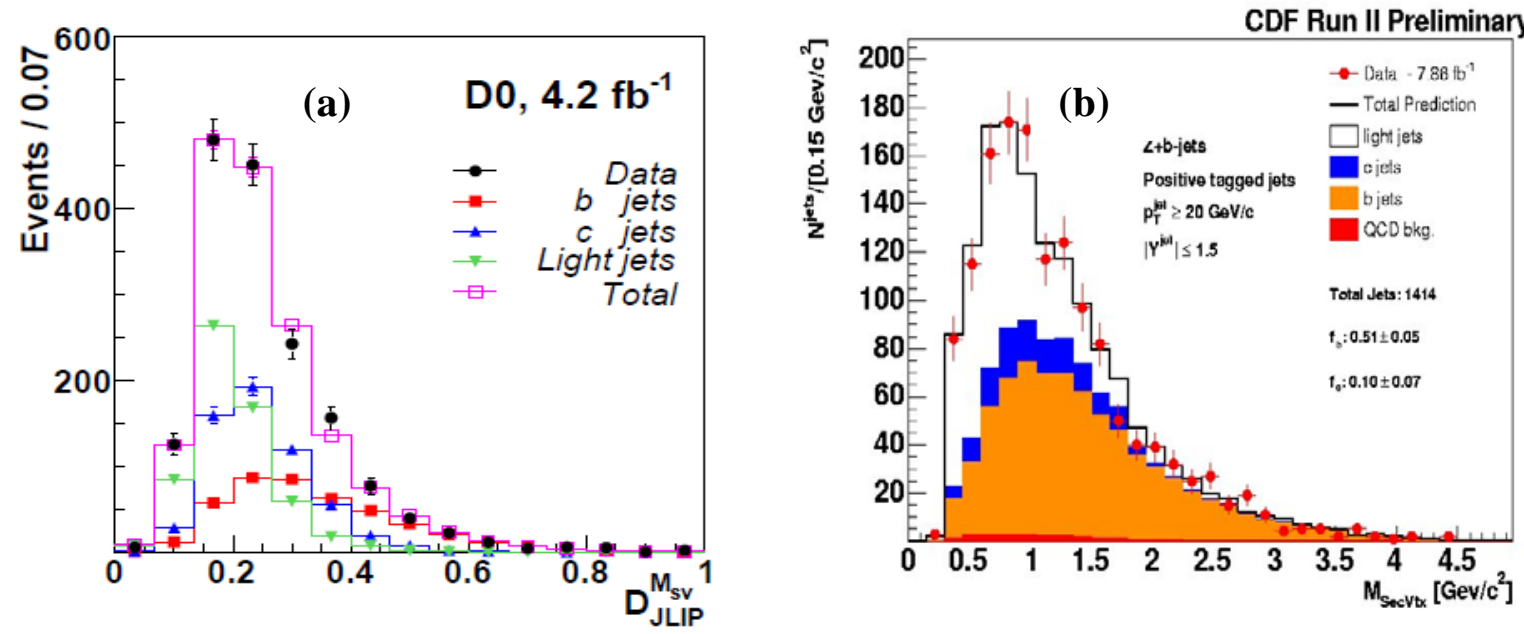

FIGURE 5. The distributions of the discriminant for $b, c$, light jets and data; MC templates are weighted by the fractions found from the fit to data. (b) The secondary vertex mass fit for the tagged jets in the selected sample.

Using $4.3 \mathrm{fb}^{-1}$ of data, the CDF has performed the first measurement of the $\mathrm{W}+$ single charm production cross section, $\sigma(\mathrm{Wc})$, using soft electron tagging [14]. The $\mathrm{W}+\mathrm{c}$ signal is mainly produced by strange quark and gluon fusion. Thus Wc production is sensitive to the s-quark content of the proton at large $\mathrm{Q}^{2}$. The $\mathrm{W}$ boson is selected via a high- $\mathrm{p}_{\mathrm{T}}$ electron and large missing $\mathrm{E}_{\mathrm{T}}$. Jets of cone size $\mathrm{R}=0.4$ are selected with $\mathrm{p}_{\mathrm{T}}>20 \mathrm{GeV}$ and $|\eta|<1.5$. Charm jets are identified from their semi-leptonic decay by looking for a electron within the jet. The measurement exploits the charge correlation between the $\mathrm{W}$ boson and the electron from the semi-leptonic decay, extracting the signal from a background-dominated sample using the excess of oppositely charged over same charge events. This yields a value $\sigma(\mathrm{Wc}) \times \mathrm{BR}=33.7 \pm 11.4$ (stat) \pm 7.3 (syst) pb which is in agreement with the NLO prediction of $16.5 \pm 4.7 \mathrm{pb}$ considering experimental and theoretical uncertainties.

\section{ACKNOWLEDGEMENT}

I would like to thank the organizers for their kind invitation to the conference. I would also like to thank the conveners of the CDF and D0 QCD groups for their help and feedback.

\section{REFERENCES}

1. D. Acosta et al. (CDF Collaboration), Phys. Rev. D 71, 032001 (2005).

2. V. M. Abazov et al. (D0 Collaboration), Nucl. Instrum. Methods Phys. Res. Sec. A 565, 463 (2006).

3. V. M. Abazov et al. (D0 Collaboration), Phys. Lett. B 693, 531 (2010).

4. T. Aaltonen et al. (CDF Collaboration), Phys. Rev. D 79, 112002 (2009).

5. V. M. Abazov et al. (D0 Collaboration), arXiv:1104.1986 (2011).

6. T. Aaltonen et al. (CDF Collaboration), arXiv:1106.5952 (2011).

7. T. Aaltonen et al. (CDF Collaboration), CDF Public Note 10216.

8. T. Aaltonen et al. (CDF Collaboration), CDF Public Note 10394.

9. V. M. Abazov et al. (D0 Collaboration), arXiv:1106.1457 (2011).

10. C. F. Berger et al., arXiv:0905.2735 [hep-ph].

11. R. K. Ellis et al., J. High Energy Phys. 01, 012 (2009); W. T. Giele and G. Zanderighi, J. High Energy Phys. 06, 038 (2008).

12. V. M. Abazov et al. (D0 Collaboration), Phys. Rev. D 83, 031105 (2011).

13. T. Aaltonen et al. (CDF Collaboration), CDF Public Note 10594.

14. T. Aaltonen et al. (CDF Collaboration), CDF Public Note 10089. 